\title{
Modeling Piezocomposite Actuators Embedded in Slender Structures
}

\author{
Carlos E.S. Cesnik* and Rafael Palacios ${ }^{\dagger}$ \\ Department of Aerospace Engineering \\ The University of Michigan, Ann Arbor, Michigan
}

\begin{abstract}
This work presents a comprehensive methodology for dimensional reduction of anisotropic slender structures with embedded anisotropic piezocomposite materials. The analysis is based on a variational-asymptotic formulation, and provides cross-sectional stiffness, inertia and actuation forces for a beam modeling of the structure. It can retain higher-order information corresponding to non-classical deformation modes. The particular case of the active anisotropic Timoshenko-like beam formulation is considered separately. Some results for typical active beam configurations are included and compared with three-dimensional shell finite element solutions using thermal analogy for the distributed actuation.
\end{abstract}

\section{Introduction}

Numerical simulation of structures through threedimensional (3-D) Finite Element Analysis has become widely accessible to engineers using the power of modern computers. However, one should not underestimate the value of engineering understanding of physical problems to identify essential features that could simplify their mathematical models. Such approach can improve the feasibility and efficiency of the structural analysis, particularly within the context of a multidisciplinary design environment.

This work presents one of such particular scenarios: slender active structures, defined as systems with one dominant spatial dimension; typical examples in aircraft applications are helicopter rotor blades and high-aspectratio wings. The basic feature of these systems is that their structural modeling process can be significantly simplified through the dimensional reduction of the 3-D structure to a (1-D) beam model

One can clearly distinguish two steps in a general beam theory [17]: first is the process of dimensional reduction, which is performed through a 2-D analysis in the beam cross sections; second, the computed equivalent stiffness and inertia properties are used in the 1-D analysis of the loaded beam. Finally, the 3-D stress/strain and displacement fields can be determined based on the

* Associate Professor. Associate Fellow, AIAA (cesnik@umich.edu)

† Graduate Research Assistant. F.X. Bagnoud Fellow (rpalacio@umich.edu) Copyright (C) 2003 by Carlos E.S. Cesnik and Rafael Palacios. Published by the American Institute of Aeronautics and Astronautics, Inc., with permission. combination of these two previous steps. This paper focuses on the dimensional reduction of general nonhomogeneous anisotropic active beams with arbitrary cross sections and initial pre-twist and curvature.

The concept of active beam refers here to beams with distributed actuators embedded within the composite structure. The actuation is assumed to derive from the electroelastic response of piezoelectric-based materials. Typical anisotropic piezocomposite actuators are the active fiber composites described by Bent [1], and the macro fiber composites of Wilkie et al. [20]. Notice that the present work encompasses also hygrothermal effects in a composite beam constitutive relation.

Several different formulations have been proposed for the analysis of anisotropic beams, among the leading efforts are the works of Giavotto et al. [10], Kosmatka [13] and Cesnik and Hodges [4]. The formulation proposed in [4], known as the Variational-Asymptotic Beam CrossSectional (VABS) analysis, will set the basic framework for this active beam development. VABS is the application to anisotropic beams of the variationalasymptotic method of Berdichevsky [2], which is based on the asymptotic solution of the 3-D cross-sectional warping field corresponding to a set of general 1-D strain measures through the minimization of the associated strain energy.

Asymptotic solutions of anisotropic beam cross sections have been pursued in the last decade by the first author and his co-workers. Successive contributions have presented the solution to the passive problem for prismatic beams [12], initially curved and twisted beams [5], beams with non-perpendicular cross-sectional planes [15], beams with arbitrary deformation modes [7], beams with transverse shear effects [14], [21], and, more recently, active cross-sectional formulation for thinwalled beams [8] and general beams [6]. In particular, the latter is restricted to a classical beam formulation (EulerBernoulli-like), which, although sufficient for most applications, does not address short composite beams (where the slenderness property is not only associated with its dimensions but also with the material anisotropy in the structure) and the estimation of the net shear loads induced by certain bending actuation modes. Finally, of practical interest is the capability to model general deformations that are not included in the classical or Timoshenko-like formulations, e.g., airfoil camber 
deformation in a slender wing. These deformation modes would require additional beam degrees of freedom.

This paper presents the most general active beam crosssectional analysis within the VABS framework. The formulation accounts for arbitrary cross-sectional geometries without restrictions in the active and passive material distribution. It also includes the effect of initial twist and curvature. Finally, finite-section effects, which can require user-defined deformation modes beyond the four classical ones (extension, twist, and two bending modes), are included in the formulation. Special attention is given to the Timoshenko-like formulation and the calculation of the active forcing constants that are part of the active beam constitutive relation. The methodology presented here has been implemented in a computer code named UM/VABS.

\section{Active VABS Formulation}

Starting with the 3-D kinematics, the cross-sectional internal energy density of the active beam is computed. Without loss of generality, the elastic component of this energy density can be written in terms of a set of 1-D measures of deformation and a warping field. The latter will act as a residual error that absorbs the difference between the 1-D and the 3-D displacement fields. In VABS formulation the cross-sectional warping is not assumed but calculated through an asymptotic expansion on the characteristic cross-sectional length $h$, which is regarded as the small parameter in the problem. The warping field will be finally solved as a function of the 1$\mathrm{D}$ measures by minimization of the energy density.

The formulation is based on the assumption of small local rotation and strain, as defined in [9], although this does not imply a restriction in the global rotations and displacements of the beam. So, a geometrically nonlinear beam theory can be constructed starting with this crosssectional formulation, as done in the exact intrinsic equations of rotating beams of Hodges [11], or in the strained-based nonlinear active beam model of Cesnik and Brown [3]. The active VABS formulation also assumes that the electroelastic constitutive relations are linear, and that the electric field is prescribed and therefore independent of the state of the electroelastic system.

\section{3-D Beam Kinematics. Strain Field}

The starting point for the VABS analysis is an accurate 3$\mathrm{D}$ formulation of the kinematics of beams undergoing deformation. The basic deformation process is shown in Figure 1. Here the results of the work of Danielson and Hodges [10] are used, where exact expressions for the 3D kinematics are given for the Jaumann-Biot-Cauchy strain tensor, $\Gamma$. Under the assumption of small local rotation, the components of this tensor in the undeformed beam reference triad $\left\{\boldsymbol{b}_{i}\right\}$ are given by:

$$
\Gamma_{i j}=\frac{1}{2}\left(A_{i j}+A_{j i}\right)-\delta_{i j}
$$

$A_{i j}$ are the components of the deformation gradient tensor, A, resolved along mixed bases:

$$
A_{i j}=\left(\boldsymbol{B}_{\boldsymbol{i}} \cdot \boldsymbol{G}_{\boldsymbol{k}}\right)\left(\boldsymbol{g}^{\boldsymbol{k}} \cdot \boldsymbol{b}_{\boldsymbol{j}}\right)
$$

$\left\{\boldsymbol{B}_{i}\right\}$ is the deformed beam reference triad, $\left\{\boldsymbol{g}^{\boldsymbol{k}}\right\}$ is the contravariant form of the tangent base vectors to the undeformed beam, and $\left\{\boldsymbol{G}_{\boldsymbol{k}}\right\}$ is the covariant base of the deformed beam.

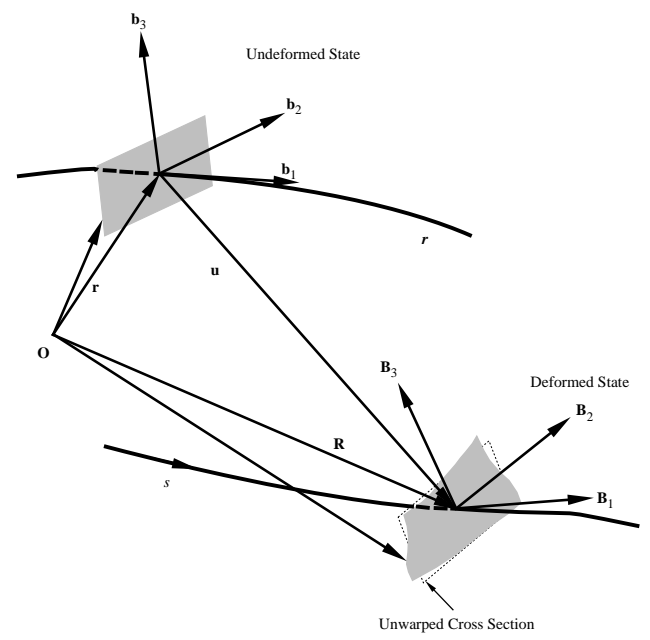

Figure 1. Kinematics of a beam

The kinematics of the reference line are set with four strain measures: the axial force strain measure, $\gamma_{11}$, and the three moment strains measures, $\kappa_{i}$, defined as the difference between the components in the curvature vector of the deformed $(\mathbf{K})$ and undeformed $(\mathbf{k})$ beam in their respective basis:

$$
\kappa_{i}=\boldsymbol{K} \cdot \boldsymbol{B}_{\boldsymbol{i}}-\boldsymbol{k} \cdot \boldsymbol{b}_{\boldsymbol{i}}
$$

From this on, the strain measures will be represented by the column vector $\in$ :

$$
\in=\left\{\gamma_{11}\left(x_{1}\right), h \kappa_{i}\left(x_{1}\right)\right\}^{T}
$$

$h$ is the characteristic length of the cross section, and it is used to non-dimensionalize the curvatures. Now, the six independent components of the strain tensor (1) can be expressed over the cross section as a function of the strain measures of equation (4), and the local value of the warping field, $v_{i}=v_{i}\left(x_{1}, x_{2}, x_{3}\right)$ :

$$
\begin{aligned}
& \Gamma\left(x_{1}, x_{2}, x_{3}\right)=\left\{\Gamma_{11}, 2 \Gamma_{12}, 2 \Gamma_{13}, \Gamma_{22}, 2 \Gamma_{23}, \Gamma_{33}\right\}^{T}= \\
& =h^{-1} \Gamma_{h} v+\Gamma_{\epsilon} \in\left(x_{1}\right)+\Gamma_{R} v+\Gamma_{l} \frac{d v}{d x_{1}}
\end{aligned}
$$

where the following matrix operators are defined as

$$
\Gamma_{h}^{T}=\left[\begin{array}{cccccc}
0 & \frac{\partial}{\partial \zeta_{2}} & \frac{\partial}{\partial \zeta_{3}} & 0 & 0 & 0 \\
0 & 0 & 0 & \frac{\partial}{\partial \zeta_{2}} & \frac{\partial}{\partial \zeta_{3}} & 0 \\
0 & 0 & 0 & 0 & \frac{\partial}{\partial \zeta_{2}} & \frac{\partial}{\partial \zeta_{3}}
\end{array}\right]
$$




$$
\begin{aligned}
& \Gamma_{\epsilon}^{T}=g^{-1 / 2}\left[\begin{array}{cccccc}
1 & 0 & 0 & 0 & 0 & 0 \\
0 & -\zeta_{3} & \zeta_{2} & 0 & 0 & 0 \\
\zeta_{3} & 0 & 0 & 0 & 0 & 0 \\
-\zeta_{2} & 0 & 0 & 0 & 0 & 0
\end{array}\right] \\
& \Gamma_{R}=g^{-1 / 2}\left[\widetilde{k}+k_{1} I_{3}\left(x_{3} \frac{\partial}{\partial x_{2}}-x_{2} \frac{\partial}{\partial x_{3}}\right)\right] \\
& 0_{3} \\
& \Gamma_{l}=g^{-1 / 2}\left[\begin{array}{c}
I_{3} \\
0_{3}
\end{array}\right]
\end{aligned}
$$

$L$ is the minimum wavelength of the beam deformation, $R$ is the typical value of the initial twist or curvature, $\zeta_{2}=x_{2} / h$ and $\zeta_{3}=x_{3} / h$ are the non-dimensional crosssectional coordinates, and the $\sim$ operator transforms a column vector to its dual skew-symmetric matrix. For this case, the metric determinant, $g$, is a function of the initial bending curvatures of the beam:

$$
\sqrt{g}=1-x_{2} k_{3}+x_{3} k_{2}
$$

The small parameters for this problem are $h / L<<1$, and $h / R<<1$, as well as the beam strains, $\in$. Further discussion on this topic can be found in [4].

\section{Cross-sectional internal energy of an active beam}

The internal energy density per unit length of an active beam is given by the combination of the strain energy density and the work produced by an electric field, $\varepsilon$. The resulting expression can be written as in [6]:

$$
2 U=\left\langle\mathrm{Z}^{T} \Gamma\right\rangle-\left\langle\Delta^{T} \varepsilon\right\rangle
$$

where following operator is defined for integration in the cross section:

$$
\bullet=\int_{S} \bullet \sqrt{g} d x_{2} d x_{3}=h^{2} \int_{S} \bullet \sqrt{g} d \zeta_{2} d \zeta_{3}
$$

$\mathrm{Z}$ and $\Delta$ are the conjugated force vectors corresponding to $\Gamma$ and $\varepsilon$, that is, the Jaumann stress vector and the local electrical displacement, respectively. Two assumptions are considered for the constitutive electro-elastic relations: 1) they are linear; 2) the elastic constants, $D$, are independent of the electrical field and the electrical constants, $E$, are independent of the deformation. That yields the simple expression (10), where superscripts refer to assumption 2).

$$
\left[\begin{array}{l}
\mathrm{Z} \\
\Delta
\end{array}\right]=\left[\begin{array}{cc}
D^{\varepsilon} & -e^{T} \\
e & \mathrm{E}^{\Gamma}
\end{array}\right] \cdot\left[\begin{array}{l}
\Gamma \\
\varepsilon
\end{array}\right]
$$

The cross-section internal energy can then be written as:

$$
2 U=\left\langle\Gamma^{T} D \Gamma\right\rangle-2\left\langle\Gamma^{T} e \varepsilon\right\rangle-\left\langle\varepsilon^{T} E \varepsilon\right.
$$

Due to the dependency set in (5), the energy will be a function of the average strain, $\in$, the applied electrical field, $\varepsilon$, and the warping field $v_{i}=v_{i}\left(x_{1}, x_{2}, x_{3}\right)$. The latter is now discretized using finite elements, so it will be written in the form of a product of a matrix of assumed shape functions $\boldsymbol{S}\left(x_{2}, x_{3}\right)$ and a vector of unknown nodal warping displacements $\boldsymbol{V}\left(x_{1}\right)$ :

$$
v_{i}\left(x_{1}, x_{2}, x_{3}\right)=S_{i j}\left(x_{2}, x_{3}\right) V_{j}\left(x_{1}\right)
$$

In the same way, one can define a known shape function related to the electric field. In this work we will assume that this electric field is unique at each cross section and that its variations along the beam are negligible with respect to the variations of the elastic variables. The generalization of the first of both assumptions to the case of several electric lines acting on different regions of the cross section is obvious.

$$
\varepsilon\left(x_{1}, x_{2}, x_{3}\right)=\Sigma\left(x_{2}, x_{3}\right) \varphi\left(x_{1}\right)
$$

After integration in the cross section, the internal energy density can be written as:

$$
\begin{aligned}
& 2 U=\epsilon^{T} D_{\in \in} \in+h^{-2} V^{T} E V+V^{T} D_{R R} V+ \\
& +V^{\prime T} D_{l l} V^{\prime}+2 h^{-1} V^{T} D_{h \in} \in+2 h^{-1} V^{T} D_{h R} V+ \\
& +2 h^{-1} V^{T} D_{h l} V^{\prime}+2 V^{T} D_{R \in} \in+2 V^{T} D_{R l} V^{\prime}+ \\
& +2 V^{\prime T} D_{l \in} \in-\varphi D_{v v} \varphi-2 \in^{T} D_{\in v} \varphi- \\
& -2 h^{-1} V^{T} D_{h v} \varphi-2 V^{T} D_{R v} \varphi-2 V^{\prime T} D_{l v} \varphi
\end{aligned}
$$

where these matrices appear during integration:

$$
\begin{array}{ll}
D_{\epsilon \in}=\left\langle\Gamma_{\epsilon}^{T} D \Gamma_{\epsilon}\right\rangle & E=\left\langle\left(\Gamma_{h} S\right)^{T} D \Gamma_{h} S\right\rangle \\
D_{R R}=\left\langle\left(\Gamma_{R} S\right)^{T} D \Gamma_{R} S\right\rangle & D_{l l}=\left\langle\left(\Gamma_{l} S\right)^{T} D \Gamma_{l} S\right\rangle \\
D_{h \epsilon}=\left\langle\left(\Gamma_{h} S\right)^{T} D \Gamma_{\epsilon}\right\rangle & D_{R \epsilon}=\left\langle\left(\Gamma_{R} S\right)^{T} D \Gamma_{\epsilon}\right\rangle \\
D_{h R}=\left\langle\left(\Gamma_{h} S\right)^{T} D \Gamma_{R} S\right\rangle & \left.D_{R l}\left(\Gamma_{R} S\right)^{T} D \Gamma_{l} S\right\rangle \\
D_{h l}=\left\langle\left(\Gamma_{h} S\right)^{T} D \Gamma_{l} S\right\rangle & D_{l \epsilon}=\left\langle\left(\Gamma_{l} S\right)^{T} D \Gamma_{\epsilon}\right\rangle \\
D_{v v}=\left\langle\Sigma^{T} E \Sigma\right\rangle & D_{h v}=\left\langle\left(\Gamma_{h} S\right)^{T} e \Sigma\right\rangle \\
D_{\epsilon v}=\left\langle\Gamma_{\epsilon}^{T} e \Sigma\right\rangle & D_{R v}=\left\langle\left(\Gamma_{R} S\right)^{T} e \Sigma\right\rangle \\
D_{l v}=\left\langle\left(\Gamma_{l} S\right)^{T} e \Sigma\right. &
\end{array}
$$

At this point the values of the beam strains $(\epsilon)$ and the applied electrical field $(\varphi)$ will be taken as independent variables in the cross-sectional problem, and the warping field will be calculated by a minimization of this internal energy. This will be possible through the variational asymptotic method due to Berdichevsky [2].

\section{Asymptotic solution for the warping field}

The unknown warping field is expressed in the form of an asymptotic expansion in the small parameter (it will be denoted as $h$, without considering its dimension). The expansion up to $\mathrm{N}$-th order perturbation terms is:

$$
V=\sum_{n=0}^{N} h^{n} V_{n}+O\left(h^{N+1}\right)
$$

Substituting (16) in (14) and keeping terms up to secondorder in $h$, the asymptotic expansion of the internal energy can be symbolically written as follows: 


$$
h^{2} U=U_{0}\left(V_{0}\right)+\sum_{n=1}^{4} h^{n} U_{n}\left(V_{0}, \ldots, V_{n} ; V_{0}^{\prime}, \ldots, V_{n-1}^{\prime} ; \in, \varphi\right)+O\left(h^{5}\right)
$$

Note that an asymptotic expansion up to $4^{\text {th }}$ order was necessary for the warping field. However, it will be seen below that the condition of minimum energy will cancel the contributions of all warping terms of order higher than 2 in (17).

To simplify the expressions, the following notation will be used for the symmetric and anti-symmetric matrices derived from the matrices in (15):

$$
2 D_{A B s}=D_{A B}+D_{B A} \quad 2 D_{A B a}=D_{A B}-D_{B A}
$$

\section{1) Classical Solution}

The classical solution for a beam is defined in terms of the four strain measures of the beam reference line given in (4), which corresponds to the theoretical beam with negligible cross-sectional area (interior problem). For the active cross section, the cross-sectional value of the electric field is added to this set of independent variables. The warping can be explicitly solved for those deformations using these assumptions:

1. The warping field is at least one order less than the strains $\in$, i.e., $\mathrm{V}_{0}=0$.

2. The warping field is orthogonal to the kernel of the matrix E, $\Psi_{c l}$, what has the physical meaning of removing from the warping its components in the rigid-body motions of the beam (three translations and the in-plane rotation). This condition can be formulated as:

$V^{T} H \Psi_{c l}=0$

$H=\left\langle S^{T} S\right.$

Definition of the kernel of a matrix implies the following conditions for $\Psi_{c l}$ :

$$
\begin{aligned}
& E \Psi_{c l}=0 \\
& \Psi_{c l}^{T} H \Psi_{c l}=I
\end{aligned}
$$

Equation (21) is necessary to normalize the column vectors of $\Psi_{c l}$. The even terms in the expansion of the energy (17), together with the former constraints, will set the necessary conditions for a recursive solution in the components of the warping.

First-order solution. $\mathrm{V}_{1}$ is the only term required to get an asymptotically correct stiffness matrix up to order $h$.

The resulting Euler equation is

$$
E V_{1}+D_{h \in} \in-D_{h v} \varphi=H \Psi_{c l} \mu_{1}
$$

A matrix of Lagrange multipliers, $\mu_{l}$, was introduced to enforce condition (19). $\mu_{1}$ is computed pre-multiplying (22) by $\Psi_{c l}^{T}$ and using relations (20). After solving for the Lagrange multipliers, equation (22) becomes

$$
E V_{1}=-\left(I-H \Psi_{c l} \Psi_{c l}^{T}\right) \cdot\left\{D_{h \in} \in-D_{h v} \varphi\right\}
$$

The matrix $E$ is four-times singular and therefore noninvertible. However, condition (19) on the warping enforces orthogonality to the kernel of $E$ and the system is solvable. The definition of a pseudo-inverse matrix $E^{+}$ with the following properties will help for this purpose:

$$
\begin{aligned}
& E E_{c l}^{+}=I-H \Psi_{c l} \Psi_{c l}^{T} \\
& E_{c l}^{+} E=I-\Psi_{c l} \Psi_{c l}^{T} H \\
& E_{c l}^{+} E E_{c l}^{+}=E_{c l}^{+}
\end{aligned}
$$

The solution can be now expressed as follows:

$$
\begin{gathered}
V_{1}=V_{1 \epsilon} \in-V_{1 \varphi} \varphi \\
V_{1 \epsilon}=-E_{c l}^{+} D_{h \epsilon} \\
V_{1 \varphi}=-E_{c l}^{+} D_{h v}
\end{gathered}
$$

Second-order solution. This term is required to recover the asymptotically correct 3-D stress/strain field of equation (6) up to order h. It is also required for the second-order expansion of the stiffness matrix. The Euler equation is:

$$
\begin{aligned}
& E V_{2}+D_{R \in} \in+2 D_{h R s} V_{1}+2 D_{h l a} V_{1}^{\prime}-D_{l \epsilon} \epsilon^{\prime}- \\
& -D_{R v} \varphi+D_{l v} \varphi^{\prime}=H \Psi_{c l} \mu_{2}
\end{aligned}
$$

Once the Lagrange multiplier $\mu_{2}$ is solved, it results in

$$
\begin{aligned}
& E V_{2}=-\left(I-H \Psi_{c l} \Psi_{c l}^{T}\right) \cdot\left\{D_{R \in} \in-D_{l \in} \in_{0}^{\prime}+\right. \\
& \left.+2 D_{h R s} V_{1}+2 D_{h l a} V_{1}^{\prime}-D_{R v} \varphi+D_{l v} \varphi^{\prime}\right\}
\end{aligned}
$$

In symbolic form, it can be written as:

$$
\begin{gathered}
V_{2}=V_{2 \epsilon} \in+V_{2 \epsilon^{\prime}} \in{ }^{\prime}-V_{2 \varphi} \varphi-V_{2 \varphi^{\prime}} \varphi^{\prime} \\
V_{2 \epsilon}=-E_{c l}^{+}\left(D_{R \epsilon}+2 D_{h R s} V_{1 \epsilon}\right) \\
V_{2 \epsilon^{\prime}}=-E_{c l}^{+}\left(-D_{l \epsilon}+2 D_{h l a} V_{1 \epsilon}\right) \\
V_{2 \varphi}=-E_{c l}^{+}\left(D_{R v}+2 D_{h R s} V_{1 \varphi}\right) \\
V_{2 \varphi^{\prime}}=-E_{c l}^{+}\left(-D_{l v}+2 D_{h l a} V_{1 \varphi}\right)
\end{gathered}
$$

\section{2) Non-classical solution with Arbitrary Modes}

Although this would be sufficient for the majority of practical cases, VABS analyses do not need to be restricted to the low-order approach of classical theories. Cross sections have a finite dimension, and the classical modes can fail to reproduce their actual deformation with a valid approximation. Here, the concept of slenderness of the beam becomes important, as well as the wavelength and frequency of the excitation or particular features such as open-cell geometry. For each particular case, a set of non-classical deformation modes of the same order of magnitude as $\in$ can be required in the 1-D model to adequately represent the 3-D behavior. A systematic approach for these effects in passive structures was done in [7] for static response and in [18] for dynamic cases. In those works, a Sturm-Liouville problem was set on the cross section, whose eigenvalues naturally yielded the non-classical deformation modes as the fundamental beam solutions. 
The approach in this paper is slightly different, in a more engineering-oriented way. The non-classical or finitesection deformation modes are assumed to be known $a$ priori from the physics of the problem. Then, a general modal formulation for the cross-sectional deformation will be presented based on a set of arbitrary finite-section modes. The formulation retains the classical modes in an explicit way, and uniqueness of the solution is imposed through orthogonality conditions. In order to include the finite-section modes in the above-introduced VABS analysis, the warping field will be divided in two terms, the external contribution of the assumed mode (in principle, same order as $\in$ ) and the remaining warping. It can be written as follows:

$$
V(x)=\Psi_{q} q(x)+W(x)
$$

$\Psi_{q}$ is the column matrix of (known) finite-section deformation modes, $q$ is the vector of their unknown magnitudes, and $W$ is the remaining unknown part of the warping field. In order to assure a consistent theory, one must impose several orthogonality conditions:

$$
\begin{aligned}
& \Psi_{q}^{T} H \Psi_{q}=I \\
& \Psi_{q}^{T} H \Psi_{c l}=0 \\
& W^{T} H \Psi_{u}=0 \text { and } \Psi_{u}=\left\lfloor\Psi_{c l} \Psi_{q}\right\rfloor
\end{aligned}
$$

$H$ and $\Psi_{c l}$ where defined in (19) and (20) and $\Psi_{u}$ is the extended matrix of deformation modes. The last condition can be rewritten as:

$$
V^{T} H \Psi_{u}=q^{T} \Psi_{q}^{T} H \Psi_{u}
$$

\section{Zero $^{\text {th }}$ - order solution}

The zero ${ }^{\text {th }}$-order component of the warping field, $V_{0}$, is obtained from the minimization of the higher order components of the energy with the constraint given by (31). If $\mu_{0}$ are the Lagrange multipliers for this constraint, the resulting Euler equation is given by

$$
E V_{0}=H \Psi_{u} \mu_{0}
$$

The solution to this equation shows that the zero ${ }^{\text {th }}$-order warping is only due to the additional modes:

$$
V_{0}=\Phi_{q} q
$$

and the matrix $\Phi_{q}$ is obtained recursively from the following equations, where $\Phi_{q}^{0}$ and $\Lambda_{q}$ are auxiliary matrices for the intermediate steps,

$$
\begin{aligned}
& E \Phi_{q}=\left(I-H \Psi_{c l} \Psi_{c l}^{T}\right) \cdot H \Psi_{q} \Lambda_{q} \\
& \Lambda_{q}{ }^{T} \Phi_{q}^{0^{T}} H \Psi_{q}=I \\
& E \Phi_{q}^{0}=\left(I-H \Psi_{c l} \Psi_{c l}^{T}\right) \cdot H \Psi_{q}
\end{aligned}
$$

\section{First-order solution}

Once $V_{0}$ is known, the first-order component, $V_{l}$, can be computed from the $h^{0}$ terms in the energy and the orthogonality conditions (30). If $\mu_{1}$ are now the Lagrange multipliers, the Euler equation for $V_{l}$ is:

$$
E V_{1}+D_{h \in} \in+2 D_{h R s} V_{0}+2 D_{h l a} V_{0}^{\prime}-D_{h v} \varphi=H \Psi_{u} \mu_{1}
$$

Once $\mu_{l}$ is eliminated, this yields:

$$
\begin{aligned}
& E V_{1}=-\left(I-H \Psi_{c l} \Psi_{c l}^{T}\right) \cdot\left(I-H \Psi_{q} \Phi_{q}^{T}\right) . \\
& \cdot\left\{D_{h \in} \in+2 D_{h R s} V_{0}+2 D_{h l a} V_{0}^{\prime}-D_{h v} \varphi\right\}
\end{aligned}
$$

Defining a pseudo-inverse matrix $E_{u}^{+}$with properties analogous to (24), the solution can be symbolically written as follows:

$$
\begin{gathered}
V_{1}=V_{1 \in} \in+V_{1 q} q+V_{1 q^{\prime}} q^{\prime}-V_{1 \varphi} \varphi \\
V_{1 \in}=-E_{u}^{+} D_{h \in} \\
V_{1 q}=-E_{u}^{+} 2 D_{h R s} \Phi_{q} \\
V_{1 q^{\prime}}=-E_{u}^{+} 2 D_{h l a} \Phi_{q} \\
V_{1 \varphi}=-E_{u}^{+} D_{h v}
\end{gathered}
$$

\section{Second-order solution}

In the same way, $V_{2}$ is computed from minimizing the $h^{2}$ terms in the energy with the constraint (31). The Euler equation in $V_{2}$ with Lagrange multipliers $\mu_{2}$ is:

$$
\begin{aligned}
& E V_{2}+D_{R \in} \in-D_{l \in} \epsilon^{\prime}+D_{R R} V_{0}+2 D_{R l a} V_{0}^{\prime}-D_{l l} V_{0}^{\prime \prime}+ \\
& +2 D_{h R s} V_{1}+2 D_{h l a} V_{1}^{\prime}-D_{R v} \varphi+D_{l v} \varphi^{\prime}=H \Psi_{u} \mu_{2}
\end{aligned}
$$

As in the former case, the solution is given by:

$$
\begin{aligned}
E V_{2}= & -\left(I-H \Psi_{c l} \Psi_{c l}^{T}\right) \cdot\left(I-H \Psi_{q} \Phi_{q}^{T}\right) \cdot\left\{D_{R \in} \in-D_{l \in} \in^{\prime}\right. \\
& +D_{R R} V_{0}+2 D_{R l a} V_{0}^{\prime}-D_{l l} V_{0}^{\prime \prime}+2 D_{h R s} V_{1}+2 D_{h l a} V^{\prime}- \\
& \left.-D_{R v} \varphi+D_{l v} \varphi^{\prime}\right\}
\end{aligned}
$$

Note the dependency on the derivatives of all the variables. In particular, $V_{1}^{\prime}$ can be evaluated by direct differentiation in (37). After substitution of the previous terms in the expansion, the second-order contribution to the warping field can be computed as:

$$
\begin{aligned}
& V_{2}=V_{2 \epsilon} \in+V_{2 \epsilon^{\prime}} \in^{\prime}+V_{2 q} q+V_{2 q^{\prime}} q^{\prime}+ \\
& +V_{2 q^{\prime \prime}} q^{\prime \prime}-V_{2 \varphi} \varphi-V_{2 \varphi^{\prime}} \varphi^{\prime} \\
& V_{2 \epsilon}=-E_{u}^{+}\left(D_{R \epsilon}+2 D_{h R s} V_{1 \epsilon}\right) \\
& V_{2 \epsilon^{\prime}}=-E_{u}^{+}\left(-D_{l \epsilon}+2 D_{h l a} V_{1 \epsilon}\right) \\
& V_{2 q}=-E_{u}^{+}\left(D_{R R} \Phi_{q}+2 D_{h R s} V_{1 q}\right) \\
& V_{2 q^{\prime}}=-2 E_{u}^{+}\left(D_{R l a} \Phi_{q}+D_{h l a} V_{1 q}+D_{h R s} V_{1 q^{\prime}}\right) \\
& V_{2 q^{\prime \prime}}=-E_{u}^{+}\left(-D_{l l} \Phi_{q}+2 D_{h l a} V_{1 q^{\prime}}\right) \\
& V_{2 \varphi}=-E_{u}^{+}\left(D_{R v}+2 D_{h R s} V_{1 \varphi}\right) \\
& V_{2 \varphi^{\prime}}=-E_{u}^{+}\left(-D_{l v}+2 D_{h l a} V_{1 \varphi}\right)
\end{aligned}
$$

\section{Second-order cross-sectional energy}

Once the warping field has been solved as a function of the 1-D variables, the general form of the second-order expansion of the cross-sectional energy given in (17) can be expressed in terms of the active beam variables. The expression for this energy, using the equations for the warping (32), (35) and (38), and the constraints in (30), finally yields 


$$
\begin{aligned}
2 U= & \\
= & h^{-2}\left[V_{0}^{T} E V_{0}\right]+ \\
+ & 2 h^{-1} V_{0}^{T}\left[D_{h \in} \in+D_{h R} V_{0}+D_{h l} V_{0}^{\prime}-D_{h v} \varphi\right]+ \\
+ & h^{0}\left[\epsilon^{T} D_{\epsilon \in} \in+V_{0}^{T} D_{R R} V_{0}+V_{0}^{\prime T} D_{l l} V_{0}^{\prime}+\right. \\
& +V_{1}^{T} D_{h \epsilon} \in+2 V_{1}^{T} D_{h R s} V_{0}+2 V_{1}^{T} D_{h l s} V_{0}^{\prime}+ \\
& +2 V_{1}^{\prime T} D_{h l}^{T} V_{0}+2 V_{0}^{T} D_{R \in} \in+2 V_{0}^{T} D_{R l} V_{0}^{\prime}+ \\
& +2 V_{0}^{\prime T} D_{l \in} \in-\varphi^{T} D_{v v} \varphi-2 \epsilon^{T} D_{\epsilon v} \varphi- \\
& \left.-V_{1}^{T} D_{h v} \varphi-2 V_{0}^{T} D_{R v} \varphi-2 V_{0}^{\prime T} D_{l v} \varphi\right]+ \\
+ & 2 h^{1}\left[V_{1}^{T} D_{R R} V_{0}+V_{1}^{\prime T} D_{l l} V_{0}^{\prime}+V_{1}^{T} D_{h R} V_{1}+\right. \\
& +V_{1}^{T} D_{h l} V_{1}^{\prime}+V_{2}^{\prime T} D_{h l}^{T} V_{0}+V_{2}^{T} D_{l l}^{T} V_{0}^{\prime}+ \\
& +V_{1}^{T} D_{R \in} \in+V_{1}^{T} D_{R l} V_{0}^{\prime}+V_{1}^{\prime T} D_{R l}^{T} V_{0}+ \\
& \left.+V_{1}^{\prime T} D_{l \in} \in-V_{1}^{T} D_{R v} \varphi-V_{1}^{\prime T} D_{l v} \varphi\right]+ \\
+ & h^{2}\left[V_{2}^{T} D_{R R} V_{0}+V_{1}^{T} D_{R R} V_{1}+2 V_{2}^{\prime T} D_{l l} V_{0}^{\prime}+\right. \\
& +V_{2}^{T} D_{l l} V_{0}^{\prime \prime}+V_{1}^{\prime T} D_{l l} V_{1}^{\prime}+2 V_{2}^{T} D_{h R s} V_{1}+ \\
& +2 V_{3}^{\prime T} D_{h l}^{T} V_{0}+2 V_{3}^{T} D_{h l}^{T} V_{0}^{\prime}+2 V_{2}^{T} D_{h l s} V_{1}^{\prime}+ \\
& +2 V_{2}^{\prime T} D_{h l}^{T} V_{1}+V_{2}^{T} D_{R \in} \in+2 V_{2}^{T} D_{R l s} V_{0}^{\prime}+ \\
& +2 V_{2}^{\prime T} D_{R l}^{T} V_{0}+2 V_{1}^{T} D_{R l} V_{1}^{\prime}+2 V_{2}^{\prime T} D_{l \in} \in+ \\
& \left.+V_{2}^{T} D_{l \in} \epsilon^{\prime}-V_{2}^{T} D_{R v} \varphi-2 V_{2}^{\prime T} D_{l v} \varphi-V_{2}^{T} D_{l v} \varphi^{\prime}\right]
\end{aligned}
$$

In this expression, the terms associated with $V_{3}$ were eliminated through integration by parts. This was possible because those terms include also the zero ${ }^{\text {th }}$-order warping, $V_{0}$, which must cancel the integral in the interior solution. By doing this, one obtains a consistent asymptotic expansion of the energy. Equation (41) also applies to the classical solution, if $V_{0}$ and its derivatives are set to zero.

\section{Beam Stiffness and Actuation Constants}

The beam internal energy density given in (41) is explicitly written now as a linear quadratic operator of the active beam 1-D variables. The matrix form of this operator yields the stiffness matrix and actuation vector that will be used in the beam analysis. The final form of both matrix and vector depends on the selection of the finite-section modes and on the truncation in the longitudinal derivatives of the variables, which depends itself on the order of the asymptotic expansion.

\section{Extended Stiffness Matrix}

For the general case, the warping field can be computed as functions of the 1-D variables $\epsilon, q$ and $\varphi$. Components of the asymptotic solution are given by equations (33), (37) and (40) and they define the extended stiffness matrix (and actuation vector). The process goes through the following steps:

1. Substitution of the warping expansions in (41), keeping terms up to order $h^{2}$. This order is required to get pretwist and curvature corrections.

2. Integration by parts in the variables that are not kept in the final expression.

3. Dismiss all explicit dependencies with $\epsilon^{\prime}, \varphi^{\prime}$ and $q$ "', which are higher-order corrections with respect to the small parameter $h / L$.

The final expression can be symbolically written as:

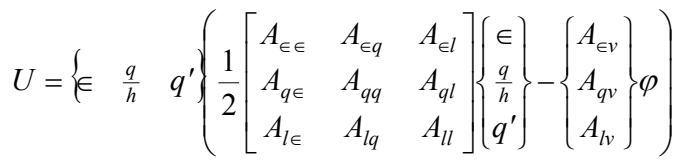

The following submatrices are defined as:

$$
\begin{array}{ll}
A_{\in l}=A_{\in q^{\prime}}-A_{\epsilon^{\prime \prime} q} & A_{q l}=A_{q q^{\prime}} \\
A_{l l}=A_{q^{\prime} q^{\prime}}-A_{q q^{\prime \prime}}-A_{q^{\prime \prime} q} & A_{v l}=A_{\varphi q^{\prime}}-A_{\varphi^{\prime} q}
\end{array}
$$

which resulted from integration by parts in the general second-order expression of the energy. Finally, after considerable simplification, the extended stiffness and actuation submatrices are given by:

$$
\begin{aligned}
& A_{\in \in}=D_{\in \in}+V_{1 \in}^{T} D_{h \in}+ \\
& +h^{1}\left[D_{R \in}^{T} V_{1 \in}+V_{1 \in}^{T}\left(D_{R \in}+2 D_{h R s} V_{1 \in}\right)\right]+ \\
& +h^{2}\left[V_{1 \epsilon}^{T} D_{R R} V_{1 €}+V_{2 €}^{T}\left(D_{R \in}+2 D_{h R s} V_{1 \in}\right)\right] \\
& A_{q q}=\Phi_{q}^{T} E \Phi_{q}+2 h^{1} \Phi_{q}^{T} D_{h R s} \Phi_{q}+ \\
& +h^{2}\left(\Phi_{q}^{T} D_{R R}+2 V_{1 q}^{T} D_{h R s}\right) \Phi_{q} \\
& A_{q \in}=\Phi_{q}^{T} D_{h \in}+h^{1}\left[\Phi_{q}^{T}\left(D_{R \in}+2 D_{h R s} V_{1 \in}\right)\right]+ \\
& +h^{2}\left[\Phi_{q}^{T} D_{R R} V_{1 \in}+V_{1 q}^{T}\left(D_{R \in}+2 D_{h R s} V_{1 \in}\right)\right] \\
& A_{q l}=\Phi_{q}^{T} D_{h l} \Phi_{q}+h^{1}\left[\Phi_{q}^{T}\left(D_{R l} \Phi_{q}+D_{h l} V_{1 q}\right)+\right. \\
& \left.+V_{1 q}^{T} D_{h l} \Phi_{q}\right]+ \\
& +h^{2}\left[\Phi_{q}^{T}\left(D_{R l} V_{1 q}+D_{R R} V_{1 q^{\prime}}+D_{h l} V_{2 q}\right)+\right. \\
& \left.+V_{1 q}^{T}\left(D_{R l} \Phi_{q}+D_{h l} V_{1 q}+2 D_{h R s} V_{1 q^{\prime}}\right)+V_{2 q}^{T} D_{h l}^{T} \Phi_{q}\right] \\
& A_{l l}=\Phi_{q}^{T}\left(D_{l l} \Phi_{q}-2 D_{h l a} V_{1 q^{\prime}}\right)+ \\
& +h\left[\Phi_{q}^{T}\left(D_{l l} V_{1 q}-2 D_{R l a} V_{1 q^{\prime}}\right)+\right. \\
& +V_{1 q}^{T}\left(D_{l l} \Phi_{q}-2 D_{h l a} V_{1 q^{\prime}}\right)+ \\
& \left.+2 V_{1 q^{\prime}}^{T}\left(D_{R l a} \Phi_{q}+D_{h l a} V_{1 q}+D_{h R s} V_{1 q^{\prime}}\right)\right] \\
& +h^{2}\left[\Phi_{q}^{T} D_{l l} V_{2 q}+V_{1 q}^{T}\left(D_{l l} V_{1 q}-2 D_{\text {Rla }} V_{1 q^{\prime}}\right)+\right. \\
& +V_{1 q^{\prime}}^{T}\left(2 D_{R l a} V_{1 q}+D_{R R} V_{1 q^{\prime}}+2 D_{h l a} V_{2 q}\right)+ \\
& +V_{2 q}^{T}\left(D_{l l} \Phi_{q}-2 D_{h l a} V_{1 q^{\prime}}\right)+ \\
& \left.+V_{2 q^{\prime}}^{T}\left(2 D_{R l a} \Phi_{q}+2 D_{h l a} V_{1 q}+2 D_{h R s} V_{1 q^{\prime}}\right)\right] \\
& A_{l \in}=\Phi_{q}^{T}\left(D_{l \in}-2 D_{h l a} V_{l \in}\right)+ \\
& +h^{1}\left[+V_{1 q}^{T}\left(D_{l \in}-2 D_{h l a} V_{1 \in}\right)+\right. \\
& \left.-2 \Phi_{q}^{T} D_{R l a} V_{1 \in}+V_{1 q^{\prime}}^{T}\left(D_{R \in}+2 D_{h R s} V_{1 \in}\right)\right]+ \\
& +h^{2}\left[-2 V_{1 q}^{T} D_{R l a} V_{1 \in}+V_{1 q^{\prime}}^{T} D_{R R} V_{1 \in}+\right. \\
& \left.+V_{2 q}^{T}\left(D_{l \in}-2 D_{h l a} V_{1 \in}\right)+V_{2 q^{\prime}}^{T}\left(D_{R \in}+2 D_{h R s} V_{1 \in}\right)\right] \\
& A_{\in v}=D_{\in v}+V_{1 \in}^{T} D_{h v}+
\end{aligned}
$$




$$
\begin{aligned}
+ & h^{1}\left[D_{R \in}^{T} V_{1 \varphi}+V_{1 \epsilon}^{T}\left(D_{R v}+2 D_{h R s} V_{1 \varphi}\right)\right]+ \\
+ & h^{2}\left[V_{1 \epsilon}^{T} D_{R R} V_{1 \varphi}+V_{2 \epsilon}^{T}\left(D_{R v}+2 D_{h R s} V_{1 \varphi}\right)\right] \\
A_{q v} & =\Phi_{q}^{T} D_{h v}+h^{1} \Phi_{q}^{T}\left(D_{R v}+2 D_{h R s} V_{1 \varphi}\right)+ \\
+ & h^{2}\left[\Phi_{q}^{T} D_{R R} V_{1 \varphi}+V_{1 q}^{T}\left(D_{R v}+2 D_{h R s} V_{1 \varphi}\right)\right] \\
A_{l v} & =\Phi_{q}^{T}\left(D_{l v}-2 D_{h l a} V_{1 v}\right)+ \\
+ & h^{1}\left[-2 \Phi_{q}^{T} D_{R l a} V_{1 \varphi}+V_{1 q}^{T}\left(D_{l v}-2 D_{h l a} V_{1 \varphi}\right)+\right. \\
& \left.+V_{1 q^{\prime}}^{T}\left(D_{R v}+2 D_{h R s} V_{1 \varphi}\right)\right]+ \\
+ & h^{2}\left[-2 V_{1 q}^{T} D_{R l a} V_{1 \varphi}+V_{1 q^{\prime}}^{T} D_{R R} V_{1 \varphi}+\right. \\
& \left.+V_{2 q}^{T}\left(D_{l v}-2 D_{h l a} V_{1 \varphi}\right)+V_{2 q^{\prime}}^{T}\left(D_{R v}+2 D_{h R s} V_{1 \varphi}\right)\right]
\end{aligned}
$$

These results can be easily simplified for the case of classical stiffness matrix with active forcing terms, by just setting to zero the terms in $q$ and $q$ ' and by using the warping field given in (25) and (28). It can also be checked the symmetry properties in matrices $A_{e e}, A_{q q}$ and $A_{l l}$. For beams without initial twist and/or curvature, all the coefficients in $h^{l}$ and $h^{2}$ are zero.

\section{Classical Second-order Stiffness Matrix}

Shear effects have a second-order contribution in beam kinematics. They can be captured as a particular case of the general solution for the extended beam kinematics presented above. An alternative approach stems from a full second-order expansion of the cross-sectional energy in the classical beam variables that is later fitted in a Timoshenko-like form. This procedure was developed for passive anisotropic beams in [14] and later modified in [21]. In this work, the formulation of [21] is extended to active beams, and this paragraph presents the classical second-order stiffness and actuation as derived from the general expression of the cross-sectional energy (41).

For classical beam theory, the zero ${ }^{\text {th }}$-order term in the warping, $V_{0}$, is set to zero. Then, the components $V_{l}$ and $V_{2}$ in the cross-sectional internal energy (41) are written as functions of the 1-D variables, $\epsilon, \epsilon^{\prime}, \varphi$ and $\varphi^{\prime}$, using equations (25) and (28). After integration by parts to remove the dependency in the derivatives of the electric field, the resulting expression for the energy in terms of beam variables is written as:

$$
\begin{aligned}
& U=\frac{1}{2} \in^{T} \quad A_{\epsilon \in} \in+h \in^{T} \quad A_{\in \epsilon^{\prime}} \in^{\prime}+ \\
& +\frac{1}{2} h^{2} \in^{\prime T} A_{\epsilon^{\prime} \epsilon^{\prime}} \in \epsilon^{\prime}+h^{2} \in^{T} A_{\in \epsilon^{\prime \prime}} \in{ }^{\prime \prime}- \\
& -\left(\epsilon^{T} A_{\epsilon v}+h \epsilon^{\prime T} A_{\epsilon^{\prime} v}+h^{2} \in^{\prime \prime T} A_{\epsilon^{\prime \prime} v}\right) \varphi
\end{aligned}
$$

where,

$$
\begin{aligned}
& A_{\epsilon^{\prime} v}=A_{\epsilon^{\prime} \varphi}-A_{\epsilon \varphi^{\prime}} \\
& A_{\epsilon^{\prime \prime} v}=A_{\epsilon^{\prime \prime} \varphi}-A_{\epsilon^{\prime} \varphi^{\prime}}+A_{\epsilon \varphi^{\prime \prime}}
\end{aligned}
$$

And the new terms in the stiffness matrix and the forcing vector are written as:

$$
\begin{aligned}
& A_{\in \epsilon^{\prime}}=\left(V_{1 €}^{T} D_{h l}+D_{l \in}^{T}\right) V_{1 \in}+ \\
& +h^{1}\left[V_{1 \in}^{T} D_{R l} V_{1 \in}+V_{1 \in}^{T} D_{h l} V_{2 €}+\right. \\
& \left.+V_{2 \epsilon}^{T} D_{h l} V_{1 \epsilon}+D_{l \epsilon}^{T} V_{2 \epsilon}\right] \\
& A_{\epsilon^{\prime} \epsilon^{\prime}}=V_{1 \in}^{T}\left(D_{l l} V_{1 \in}+D_{h l s} V_{2 \epsilon^{\prime}}\right)+ \\
& +V_{2 \epsilon^{\prime}}^{T}\left(D_{h l s} V_{1 \epsilon}+D_{l \epsilon}\right) \\
& A_{\in \in \epsilon^{\prime \prime}}=h^{0}\left[\left(V_{1 \in}^{T} D_{h l}+D_{l \in}^{T}\right) V_{2 \epsilon^{\prime}}\right] \\
& A_{\epsilon^{\prime} v}=V_{1 \in}^{T}\left(D_{l v}+2 D_{h l a}^{T} V_{1 \varphi}\right)-D_{l \in}^{T} V_{1 \varphi}+ \\
& +h^{1}\left[-D_{l \in}^{T} V_{2 \varphi}+V_{2 \epsilon}^{T}\left(D_{l v}+2 D_{h l a}^{T} V_{1 \varphi}\right)\right. \\
& \left.+2 V_{1 \in}^{T}\left(D_{R l a}^{T} V_{1 \varphi}+D_{h l a}^{T} V_{2 \varphi}\right)\right] \\
& A_{\epsilon^{\prime \prime} v}=-V_{1 \epsilon}^{T} D_{l l} V_{1 \varphi}+V_{2 \epsilon^{\prime}}\left(D_{l v}+2 D_{h l a} V_{1 \varphi}\right)
\end{aligned}
$$

\section{Stiffness Matrix for Timoshenko-like Theory}

This active VABS solution for Timoshenko-like beams is an extension to the theory derived by $\mathrm{Yu}$ et al. [21]. Some details of the process are skipped when they are not substantially different from those presented in the passive formulation.

In an Euler-Bernoulli-like solution, the deformed beam reference triad $\left\{\boldsymbol{B}_{i}\right\}$ of Figure 1 is tangent to the deformed beam reference line. This is no longer the case when transverse shear effects are included, and the rotation angles for this shear deformation are identified in the standard Timoshenko formulation, [16], as the engineering shear strains, $\gamma_{1 \alpha}$. In addition to this, the variables in a Timoshenko-like formulation must be expressed with respect to the actual location of the deformed reference triad, and this involves a change in the definition of the moment strains (curvatures) used in the classical analysis. To do this, the following change of variables is used:

$$
\begin{aligned}
& \in=e+P \gamma+Q \gamma^{\prime} \\
& e=\left[\begin{array}{llll}
\gamma_{11} & \beta_{1} & \beta_{2} & \beta_{3}
\end{array}\right]^{T}, \gamma=\left[\begin{array}{ll}
\gamma_{12} & \gamma_{23}
\end{array}\right]^{T} \\
& P^{T}=\left[\begin{array}{cccc}
0 & k_{2} & -k_{1} & 0 \\
0 & -k_{3} & 0 & -k_{1}
\end{array}\right], Q^{T}=\left[\begin{array}{cccc}
0 & 0 & 0 & 1 \\
0 & 0 & -1 & 0
\end{array}\right]
\end{aligned}
$$

Considering that the shear strains are one order lower than the bending strains, the second-order energy (45) is transformed to

$$
\begin{aligned}
U & =\frac{1}{2} e^{T} A_{\epsilon \in} e-e^{T} A_{\epsilon \nu} \varphi+ \\
+ & h^{1}\left(e^{T} A_{\in \epsilon^{\prime}} e^{\prime}-e^{T} A_{\epsilon^{\prime} \nu} \varphi\right)+ \\
+ & h^{2}\left[e^{T} A_{\epsilon \in}\left(h^{-2} P \gamma\right)+e^{T} A_{\epsilon \in}\left(h^{-2} Q \gamma^{\prime}\right)+\right. \\
& +\frac{1}{2} e^{\prime T} A_{\epsilon^{\prime} \epsilon^{\prime}} e^{\prime}+e^{T} A_{\in \epsilon^{\prime \prime}} e^{\prime \prime}-e^{\prime \prime T} A_{\epsilon^{\prime \prime} \nu} \varphi- \\
& \left.-\left(h^{-2} \gamma^{T} P^{T}\right) A_{\epsilon \nu} \varphi-\left(h^{-2} \gamma^{\prime T} Q^{T}\right) A_{\epsilon v} \varphi\right]
\end{aligned}
$$

In active Timoshenko beam theory, the cross-sectional internal energy would be written as 


$$
\begin{aligned}
& U=\frac{1}{2} e^{T} B_{e e} e+e^{T} B_{e \gamma} \gamma+\frac{1}{2} \gamma^{T} B_{\gamma \gamma} \gamma- \\
& -\left(e^{T} B_{e v}+\gamma^{T} B_{\gamma}\right) \cdot \varphi
\end{aligned}
$$

Comparison of expressions (48) and (49) shows that the Timoshenko formulation is not asymptotically correct. Although a second-order expansion is necessary to include the shear terms, these are not the only additional contributions to the expansion. As a result, some fitting procedure is necessary to reduce equation (48) to an expression like (49). This is achieved in [21] by imposing local equilibrium of beam forces. The methodology is extended here for the active beam formulation. The linearized equilibrium equations are:

$$
\begin{aligned}
F_{e}^{\prime}+D_{3} F_{e}+D_{4} F_{\gamma}=0 & \\
F_{\gamma}^{\prime}+D_{1} F_{\gamma}+D_{2} F_{e}=0 & \\
D_{1} & =\left[\begin{array}{cc}
0 & -k_{1} \\
k_{1} & 0
\end{array}\right] \quad D_{2}=\left[\begin{array}{cccc}
k_{3} & 0 & 0 & 0 \\
-k_{2} & 0 & 0 & 0
\end{array}\right] \\
D_{3} & =\left[\begin{array}{cccc}
0 & 0 & 0 & 0 \\
0 & 0 & -k_{3} & k_{2} \\
0 & k_{3} & 0 & -k_{1} \\
0 & -k_{2} & k_{1} & 0
\end{array}\right] \quad D_{4}=Q-D_{2}^{T}
\end{aligned}
$$

$F_{e}$ and $F_{\gamma}$ are the force conjugates corresponding to the strain measures $e$ and $\gamma$. The linear electroelastic constitutive relations comes directly from equation (49), and the equilibrium equations can then be finally written in terms of the strain measures,

$$
\begin{aligned}
& {\left[\begin{array}{ll}
B_{e e} & B_{e \gamma} \\
B_{\gamma} & B_{\gamma \gamma}
\end{array}\right]\left\{\begin{array}{l}
e^{\prime} \\
\gamma^{\prime}
\end{array}\right\}+} \\
& +\left[\begin{array}{ll}
D_{3} B_{e e}+D_{4} B_{\gamma e} & D_{3} B_{e \gamma}+D_{4} B_{\gamma \gamma} \\
D_{2} B_{e e}+D_{1} B_{\gamma e} & D_{2} B_{e \gamma}+D_{1} B_{\gamma \gamma}
\end{array}\right]\left\{\begin{array}{l}
e \\
\gamma
\end{array}\right\}=0
\end{aligned}
$$

and this set of equations gives expressions for $e^{\prime}$ and $\gamma^{\prime}$ in terms of $e, \gamma$ and $\varphi$.

$$
\begin{aligned}
e^{\prime}= & L_{e^{\prime} e} e+L_{e^{\prime} \gamma} \gamma \\
\gamma^{\prime}= & L_{\gamma^{\prime} e} e+L_{\gamma^{\prime} \gamma} \gamma \\
L_{e^{\prime} e} & =N^{-1}\left(B_{e \gamma} B_{\gamma \gamma}^{-1} D_{2}-D_{3}\right) B_{e e}+ \\
& +N^{-1}\left(B_{e \gamma} B_{\gamma \gamma}^{-1} D_{1}-D_{4}\right) B_{\gamma e} \\
L_{e^{\prime} \gamma} & =N^{-1}\left(B_{e \gamma} B_{\gamma \gamma}^{-1} D_{2}-D_{3}\right) B_{e \gamma}+ \\
& +N^{-1}\left(B_{e \gamma} B_{\gamma \gamma}^{-1} D_{1}-D_{4}\right) B_{\gamma \gamma} \\
L_{\gamma^{\prime} e} & =-B_{\gamma \gamma}^{-1}\left[B_{\gamma e} L_{e^{\prime} e}+D_{1} B_{\gamma e}+D_{2} B_{e e}\right] \\
L_{\gamma^{\prime} \gamma} & =-B_{\gamma \gamma}^{-1}\left[B_{\gamma e} L_{e^{\prime} \gamma}+D_{1} B_{\gamma \gamma}+D_{2} B_{e \gamma}\right] \\
N & =B_{e e}-B_{e \gamma} B_{\gamma \gamma}^{-1} B_{\gamma e}
\end{aligned}
$$

Differentiation of the first equation in (52) gives

$$
e^{\prime \prime}=\left(L_{e^{\prime} e} L_{e^{\prime} e}+L_{e^{\prime} \gamma} L_{\gamma^{\prime} e}\right) e+\left(L_{e^{\prime} e} L_{e^{\prime} \gamma}+L_{e^{\prime} \gamma} L_{\gamma^{\prime} \gamma}\right) \gamma
$$

Results (52) and (53) allow the casting of the secondorder energy given by (48) into a Timoshenko-like matrix as in (49). If the small parameter $h$ is removed from the equations, their final form is

$$
\begin{aligned}
& B_{e e}=A_{\epsilon \in}+2\left(A_{\in \in} Q L_{\gamma^{\prime} e}\right)_{s}+2\left(A_{\epsilon \epsilon^{\prime}} L_{e^{\prime} e}\right)_{s}+ \\
& +L_{e^{\prime} e}^{T} A_{\epsilon^{\prime} \epsilon^{\prime}} L_{e^{\prime} e}+2\left[A_{\epsilon \epsilon^{\prime \prime}}\left(L_{e^{\prime} e} L_{e^{\prime} e}+L_{e^{\prime} \gamma} L_{\gamma^{\prime} e}\right)\right]_{s} \\
& B_{e \gamma}=A_{\epsilon \in}\left(P+Q L_{\gamma^{\prime} \gamma}\right)+A_{\epsilon \epsilon^{\prime}} L_{e^{\prime} \gamma}+ \\
& +L_{e^{\prime} e}^{T} A_{\epsilon^{\prime} \epsilon^{\prime}} L_{e^{\prime} \gamma}+A_{\epsilon \epsilon^{\prime \prime}}\left(L_{e^{\prime} e} L_{e^{\prime} \gamma}+L_{e^{\prime} \gamma} L_{\gamma^{\prime} \gamma}\right) \\
& B_{\gamma^{\prime}}=L_{e^{\prime} \gamma}^{T} A_{\epsilon^{\prime} \epsilon^{\prime}} L_{e^{\prime} \gamma} \\
& B_{e v}=\left(I+L_{\gamma^{\prime} e}^{T} Q^{T}\right) A_{\epsilon v}+L_{e^{\prime} e}^{T} A_{\epsilon^{\prime} v}+ \\
& +\left(L_{e^{\prime} e}^{T} L_{e^{\prime} e}^{T}+L_{\gamma^{\prime} e}^{T} L_{e^{\prime} \gamma}^{T}\right) A_{\epsilon^{\prime \prime} v} \\
& B_{w}=\left(P^{T}+L_{\gamma^{\prime} \gamma}^{T} Q^{T}\right) A_{\epsilon v}+L_{e^{\prime} \gamma}^{T} A_{\epsilon^{\prime} v}+ \\
& +\left(L_{e^{\prime} e} L_{e^{\prime} \gamma}+L_{e^{\prime} \gamma} L_{\gamma^{\prime} \gamma}\right)^{T} A_{\epsilon^{\prime \prime} v}
\end{aligned}
$$

For the stiffness submatrices, these expressions are equivalent to those given in [21], except for the symmetry properties in $B_{e e}$, which are not imposed in that work. Computing the elastic $B$ matrices for a beam with initial pretwist and curvature from this set of complex equations is not a trivial task. A solution using a perturbation scheme is presented in [21]. The last two equations are uncoupled and directly give the actuation coefficients after the stiffness matrix is known.

\section{Beam Inertia Constants}

A complete formulation of the equations of the beam dynamics requires the computation of the equivalent inertia constants corresponding to the beam cross section. In a classical or Timoshenko-like beam theory, the inertia associated with the deformation of the structure is normally neglected in comparison with the inertia of the rigid-body motions of the cross section (three translations and three rotations). This assumption leads to the conventional representation of the kinetic energy of the beam through moments of inertia.

The formulation for analysis of active beams in this work has introduced a set of non-classical deformation modes for an adequate representation of higher-order effects. A consistent formulation of the dynamics of such beams should then take into account the inertia associated with these higher-order components of the motion. This section presents such formulation in the context of the evaluation of cross-sectional properties of slender structures for their dimensional reduction.

The kinetic energy density per unit length of a slender structure is given by:

$$
2 T=\left\langle\rho \dot{r}_{\boldsymbol{P}}^{T} \dot{\boldsymbol{r}}_{\boldsymbol{P}}\right\rangle
$$

In this expression $\boldsymbol{r}_{\boldsymbol{P}}\left(t ; x_{2}, x_{3}\right)$ is the position of a point $P$ in the cross-sectional coordinates $\left(x_{2}, x_{3}\right)$ at time $t$ in a given fixed reference frame. The absolute motion of this point can be expressed as a combination of the motion of the cross-sectional reference system $\left(\boldsymbol{V}_{\boldsymbol{0}}, \boldsymbol{\Omega}_{\boldsymbol{0}}\right)$ and the local motion of the point in that system, which can be expressed as a combination of the finite-section deformation modes. 


$$
\dot{\boldsymbol{r}}_{\boldsymbol{P}}=\boldsymbol{V}_{\boldsymbol{0}}+\Omega_{\boldsymbol{0}} \times\left(\boldsymbol{r}_{\boldsymbol{P}}-\boldsymbol{r}_{\boldsymbol{0}}\right)+\boldsymbol{\psi}_{q} \dot{q}
$$

The last term in this equation includes the non-classical motion through $N$ modes. The $3 \times N$ matrix operator $\psi_{q}\left(x_{2}, x_{3}\right)$ defines the finite-section mode shapes, and their amplitudes are given by the vector $q$. This operator is the continuous version of the assumed-modes matrix $\Psi_{q}$ defined in equation (29). It satisfies then a set of integral orthogonality conditions analogous to those given in (30). The beam kinetic energy density can be computed as a function of the beam 1-D variables, after integration of equation (55) with the velocity field defined by (56). That leads to the following matrix expression

$$
T=\frac{1}{2}\left\{\begin{array}{lll}
V_{0} & \Omega_{0} & q
\end{array}\right\}\left[\begin{array}{lll}
m_{r r} & m_{r \theta} & m_{r q} \\
m_{\omega \theta} & m_{\theta \theta} & m_{\theta q} \\
m_{q r} & m_{q \theta} & m_{q q}
\end{array}\right]\left\{\begin{array}{c}
V_{0} \\
\Omega_{0} \\
q
\end{array}\right\}
$$

where,

$$
\begin{aligned}
& m_{r r}=\langle\rho \boldsymbol{I}\rangle, m_{r \theta}=\left\langle\rho\left[\begin{array}{ccc}
0 & x_{3} & -x_{2} \\
-x_{3} & 0 & 0 \\
x_{2} & 0 & 0
\end{array}\right]\right) \\
& m_{\theta \theta}=\left\langle\rho\left[\begin{array}{ccc}
x_{2}{ }^{2}+x_{3}{ }^{2} & 0 & 0 \\
0 & x_{3}{ }^{2} & -x_{2} x_{3} \\
0 & -x_{2} x_{3} & x_{2}{ }^{2}
\end{array}\right]\right) \\
& m_{q r}=\left\langle\rho\left[\begin{array}{lll}
\Psi_{q 1} & \Psi_{q 2} & \Psi_{q 3}
\end{array}\right]\right. \\
& m_{q \theta}=\left\langle\rho\left[\left(x_{2} \Psi_{q 3}-x_{3} \Psi_{q 2}\right) \quad x_{3} \Psi_{q 1}-x_{2} \Psi_{q 1}\right]\right. \\
& m_{q q}=\left\langle\rho\left[\Psi_{q 1}^{2}+\Psi_{q 2}^{2}+\Psi_{q 3}^{2}\right]\right.
\end{aligned}
$$

Here, the transpose modal matrix was decomposed into its column vectors, corresponding to the coordinates of the assumed modes, as follows:

$$
\psi_{q}^{T}=\left[\begin{array}{lll}
\Psi_{q 1} & \Psi_{q 2} & \Psi_{q 3}
\end{array}\right]
$$

Note that for the case of constant density in the cross section, the columns of $m_{q r}$ are zero and there exist a decoupling between the translational inertia and the one of the finite-section modes. This occurs as a direct consequence of the orthogonality conditions (30).

\section{Example Case: Active Composite Box-Beam}

The active beam analysis methodology described above has been implemented in a computer program named $\mathrm{UM} / \mathrm{VABS}$. This section presents numerical results design to exemplify the new capabilities of the methodology, focusing on active and non-classical beam modeling.

The test cases correspond to an active square box beam with constant properties along the beam axis, and a cross section of $25 \mathrm{~mm}$ between mid-lines. The upper and lower sides are made of four plies of AS4/3506-1 at $45^{\circ}$ with the beam axis, and the lateral sides are made of four plies of a typical anisotropic piezocomposite actuator
(APA) at $-45^{\circ}$. Actuation on the piezocomposite plies is $\pm 2000 \mathrm{~V}$. Material properties are given in Table 1 .

Table 1. Material properties in active box beam $(1=$ fiber direction, $2=$ transverse and in plane of the layer, $3=$ out-of-plane)

\begin{tabular}{ccrr} 
& & AS4/3506-1 & APA \\
\hline $\mathrm{E}_{11}$ & $(\mathrm{GPa})$ & 142 & 42.2 \\
$\mathrm{E}_{22}$ & $(\mathrm{GPa})$ & 9.8 & 17.5 \\
$\mathrm{G}_{12}$ & $(\mathrm{GPa})$ & 6.0 & 5.5 \\
$\mathrm{G}_{23}$ & $(\mathrm{GPa})$ & 4.8 & 4.4 \\
$v_{12}$ & - & 0.3 & 0.354 \\
$v_{23}$ & - & 0.42 & 0.42 \\
$\mathrm{~d}_{111}$ & $(\mathrm{~m} / \mathrm{V})$ & - & $3.81 \cdot 10^{-10}$ \\
$\mathrm{~d}_{112}$ & $(\mathrm{~m} / \mathrm{V})$ & - & $-1.6 \cdot 10^{-10}$ \\
thickness & $(\mathrm{mm})$ & 0.127 & 0.127 \\
distance & & & \\
electrodes & $(\mathrm{mm})$ & - & 1.143 \\
\hline
\end{tabular}

A 640 8-noded finite element model was created in $\mathrm{UM} / \mathrm{VABS}$ for the numerical analysis (Figure 2).

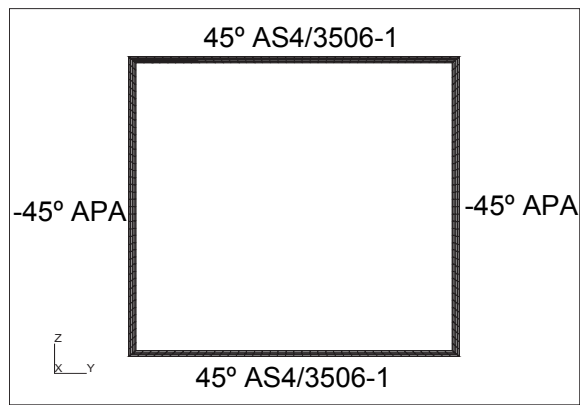

Figure 2. UM/VABS mesh for the box-beam

Finite-section modes for shear deformation.

First, results are compared for two different approaches to the dimensional reduction of active beams if shear effects are included: 1) direct use of the Timoshenko-like formulation; 2) solution assuming shear modes as finitesection deformation modes.

The general form of the non-classical formulation can approximate the solution for Timoshenko-like beams if the following modes are taken to represent the shear deformation:

$$
\begin{aligned}
& x_{1} x_{2} \text {-shear: } \Psi_{q_{1}}^{T}\left(x_{2}, x_{3}\right)=\left\{\begin{array}{lll}
x_{2} & 0 & 0
\end{array}\right\} \\
& x_{1} x_{3} \text {-shear: } \Psi_{q_{2}}^{T}\left(x_{2}, x_{3}\right)=\left\{\begin{array}{lll}
x_{3} & 0 & 0
\end{array}\right\}
\end{aligned}
$$

The corresponding stiffness matrix is then determined by the set $\left(\varepsilon, q_{1}, q_{2}, \kappa_{1}, \kappa_{2}, \kappa_{3}\right)$.

A particular feature of this selection for prismatic beams is that the derivatives of the new modes $\left(q_{1}^{\prime}, q_{2}{ }^{\prime}\right)$ are the beam bending strains. This can be shown by comparing the force equations associated with the new degrees of freedom and the bending moment equations. Therefore the first derivatives of the non-classical modes do no provide additional information and can be discarded in the dimensional reduction.

Stiffness constants and actuation forces were computed 
for two different applied electric fields, corresponding to bending $(-2000 \mathrm{~V} / 2000 \mathrm{~V})$ and twist $(2000 \mathrm{~V} / 2000 \mathrm{~V})$ actuations. The non-zero values are shown in Table 2.

Table 2. Stiffness and actuation for Box Beam

\begin{tabular}{|c|c|c|c|}
\hline & Timoshenko & $\begin{array}{c}\text { Assumed } \\
\text { modes }\end{array}$ & \%Diff \\
\hline $\mathrm{S}_{11 \times 10^{-5}}(\mathrm{~N})$ & 8.142 & 8.142 & 0.0 \\
\hline $\mathrm{S}_{14} \times 10^{-3} \quad(\mathrm{~N} \mathrm{~m})$ & -1.569 & -1.568 & -0.1 \\
\hline $\mathrm{S}_{22} \times 10^{-5} \quad(\mathrm{~N})$ & 2.547 & 2.598 & 2.0 \\
\hline $\mathrm{S}_{25} \times 10^{-3} \quad(\mathrm{~N} \mathrm{~m})$ & 1.998 & 2.043 & 2.3 \\
\hline $\mathrm{S}_{33} \times 10^{-5} \quad(\mathrm{~N})$ & 1.975 & 2.341 & 18.5 \\
\hline $\mathrm{S}_{36} \times 10^{-2} \quad(\mathrm{~N} \mathrm{~m})$ & -2.561 & -2.346 & -8.4 \\
\hline $\mathrm{S}_{44} \times 10^{-1} \quad\left(\mathrm{~N} \mathrm{~m}^{2}\right)$ & 9.238 & 9.238 & 0.0 \\
\hline $\mathrm{S}_{55} \times 10^{-1}\left(\mathrm{~N} \mathrm{~m}^{2}\right)$ & 9.573 & 9.613 & 0.4 \\
\hline $\mathrm{S}_{66 \times 10^{-1}\left(\mathrm{~N} \mathrm{~m}^{2}\right)}$ & 8.445 & 8.448 & 0.0 \\
\hline \multicolumn{4}{|l|}{ Bending Actuation: } \\
\hline $\mathrm{F}_{3}{ }^{(\mathrm{a})} \quad(\mathrm{N} / \mathrm{m})$ & 196.97 & 232.67 & 18.1 \\
\hline $\mathrm{M}_{3}^{(\mathrm{a})} \quad(\mathrm{N})$ & -1.249 & -1.283 & 2.7 \\
\hline \multicolumn{4}{|l|}{ Twist Actuation: } \\
\hline$F_{1}{ }^{(a)} \quad(N / m)$ & 17.219 & 17.219 & 0.0 \\
\hline $\mathrm{M}_{1}^{(\mathrm{a})}$ & 3.666 & 3.666 & 0.0 \\
\hline
\end{tabular}

The results indicate that the finite-section modes defined in (60) can approximate the stiffness constants and actuation within a $20 \%$ error. However, as it will be seen next, this discrepancy at the cross-sectional level does not significantly affect the 1-D results.

One-dimensional analysis: Twist actuation

Consider a 500-mm long cantilever beam with the former cross section. A 3-D shell FEM model was created using MSC.Nastran and thermal analogy was used for the piezoelectric properties. The deformed model under $-2000 \mathrm{~V} /+2000 \mathrm{~V}$ loading (corresponding to twist actuation) is shown in Figure 3.

A second analysis was performed using an in-house 1-D nonlinear finite element solver. The stiffness and actuation constants derived from the Timoshenko and the assumed modes formulations, as well as from a pure $4 \times 4$ Euler formulation are used in the 1-D analysis. The tip twist angle from the 3-D shell finite element solution is $1.195^{\circ}$, compared to $1.188^{\circ}$ obtained by the three different beam models.

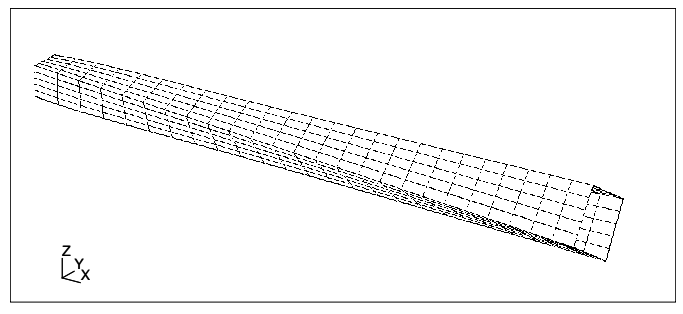

Figure 3. Deformed 3-D FEM for box-beam under twist actuation

For this configuration, the response to twist actuation is accurately reproduced for any slenderness even using an Euler-like beam model, due to absence of shear effects. Bending actuation: Timoshenko vs. Euler formulations. From general beam theory, it is known that the
Timoshenko model gives a better approximation for short beams, in which shear deformation becomes important. In long beams, applied shear loads are counteracted by bending moments, and so the Euler-Bernoulli formulation can capture their effect.

On the other hand, for an active beam, the local actuation shear resultant can only be captured by a Timoshenkolike model, regardless of the slenderness of the beam. So, a cross-sectional active Timoshenko analysis becomes necessary for all beams in which the actuation induces shear loads, even when corrections in the beam stiffness are not important.

This can be seen in the present box beam numerical test case, when the beam is actuated in bending (opposite electric fields between both sides). Table 3 summarizes the active forces per unit length based on both the Timoshenko and Euler-Bernoulli-like analyses.

\begin{tabular}{lccc}
\multicolumn{4}{c}{ Table 3. Stiffness and actuation for Box Beam } \\
\hline & & Timoshenko & Euler \\
\hline $\mathrm{F}_{3}$ & $(\mathrm{~N} / \mathrm{m})$ & 196.97 & - \\
$\mathrm{M}_{3}$ & $(\mathrm{~N} \mathrm{~m} / \mathrm{m})$ & -1.249 & -0.994 \\
\hline
\end{tabular}

The induced tip deformation for different beam slender ratios were computed using all three-beam models and compared with the 3-D shell finite element model (Figure 4). The lateral displacement was induced by the actuation moment and all solutions coincide; however, the shear force is missing in the Euler analysis and therefore the vertical displacement cannot be predicted by this formulation, while the other two matches perfectly with MSC.Nastran.
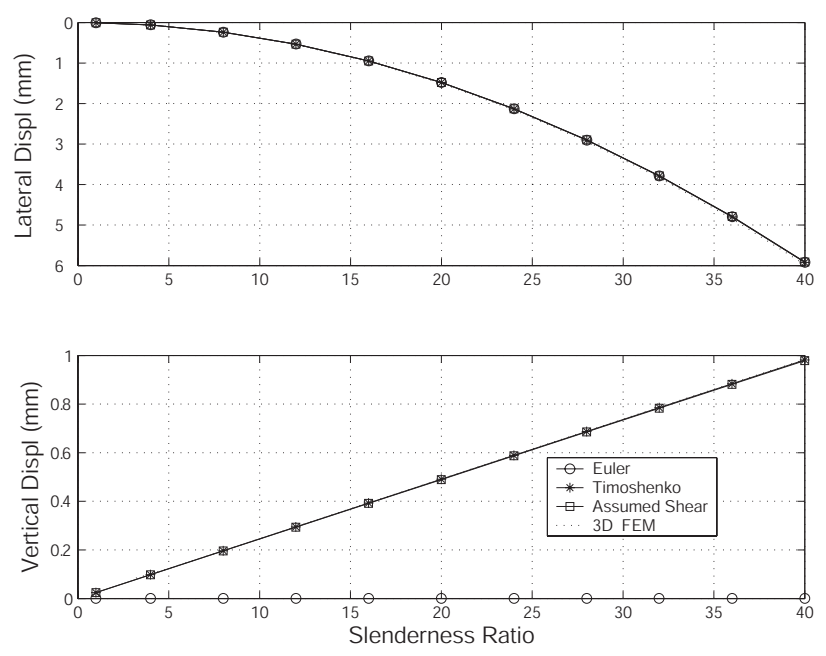

Figure 4. Box beam tip deflections under bending actuation

\section{Conclusions}

A numerical method to analyze general (thin-walled, thick-walled, or solid) non-homogeneous and nonisotropic initially twisted and curved beams with embedded anisotropic piezocomposite actuators is derived. It is an asymptotical analysis that reduces the 
original three-dimensional electroelastic formulation to a one-dimensional problem. The formulation provides the stiffness, inertia, and actuation constants for the classical extension, twist, and bending degrees of freedom as well as for non-classical deformation modes. These include Timoshenko-like theory and other higher-order formulations depending on the choice of cross-section finite deformation modes. A consistent set of actuation constants corresponding to the different formulations also results from the analysis. This provides the necessary information to analyze slender structures with embedded anisotropic piezocomposite actuators. Due to the generality of the formulation, no closed form solution exists, and the solutions are numerically obtained using the finite element method. The different formulations have been implemented in the computer code UM/VABS. A simple active structure is used to exemplify the flexibility of the formulation and the required refinement when dealing with torsion and bending actuation modes. The accuracy of the results is verified against threedimensional shell finite element representation of the test structure. Among other things, results show the importance of having transverse shear degrees of freedom when analyzing off-axis bending actuation. The induced lateral displacement caused by the section shear effects cannot be modeled by the classical Euler-Bernoulli-like theory. Finally, the example showed the finitedeformation mode method as a powerful approach to augment the classical beam kinematics.

\section{Acknowledgements}

This work was supported by the Army Research Office under grant 43854-EG. The technical monitor is Dr. Gary L. Anderson. The second author also gratefully acknowledges the scholarship received from the Fulbright Commission-Spain for part of this work.

\section{References}

[1] Bent, A. A. "Active Fiber Composite Material Systems for Structural Control Applications". Proceedings of the SPIE's $6^{\text {th }}$ International Symposium on Smart Structures and Materials, Newport Beach, CA, March 1999

[2] Berdichevsky, V.L. "On the energy of an elastic rod". PMM, Vol 45, 1982. pp.518-529

[3] Cesnik C.E.S., Brown E.L. "Modeling of High Aspect Ratio Active Flexible Wings for Roll Control". Proceedings of the $43^{\text {rd }}$ AIAA/ASME/ ASCE/AHS/ASC Structures, Structural Dynamics and Materials Conference, Denver, CO, April 2002. AIAA Paper 2002-1719

[4] Cesnik C.E.S., Hodges D.H. "VABS: "A New Concept for Composite Rotor Blade Cross-Sectional Modeling". Journal of the American Helicopter Society, Vol 42, No 1, 1997

[5] Cesnik C.E.S., Hodges D.H., Sutyrin V.G. "Cross-Sectional Analysis of Composite Beams Including Large Initial Twist and Curvature Effects". AIAA Journal, Vol 34 No 9, Sept 1996

[6] Cesnik C.E.S., Ortega-Morales M. "Active Beam CrossSectional Modeling". Journal of Intelligent Material Systems and Structures, Vol 12, No 7, July 2001. pp 483-96

[7] Cesnik C.E.S., Sutyrin V.G., Hodges D.H. "Refined Theory of Composite Beams: "The Role of Short-Wavelength Extrapolation”. International Journal of Solids and Structures, Vol 33, No 10, 1996

[8] Cesnik C.E.S., Shin S. "On the modeling of integrally actuated helicopter blades". International Journal of Solids and Structures, Vol 38 No 10-13, Mar 2001. pp. 1765-1789

[9] Danielson D.A., Hodges D.H. "Nonlinear Beam Kinematics by Decomposition of the Rotation Tensor". Journal of Applied Mechanics, Vol 54 No 2, 1987, pp. 258-262

[10] Giavotto V., Borri M., Mantegazza P., Giringhelli G., Carmaschi V., Maffioli G.C., Mussi F. "Anisotropic beam theory and applications". Computers and Structures, Vol 16, No 1-4, 1983; pp.403-413

[11] Hodges D.H. "A mixed variational formulation based on exact intrinsic equations for dynamics of moving beams". International Journal of Solids and Structures, Vol 26 No 11 1990 pp 1253-1273

[12] Hodges D.H., Atilgan A.R., Cesnik C.E.S., Fulton M.V. "On a simplified strain energy function for geometrically nonlinear behaviour of anisotropic beams". Composites Engineering, Vol 2, No 5-7, 1992

[13] Kosmatka J.B. "On the behavior of pretwisted beams with irregular cross-sections". ASME Journal of Applied Mechanics, Vol 59, No 1 Mar 1992 . pp. 146-152

[14] Popescu B., Hodges D.H. "On asymptotically correct Timoshenko-like anisotropic beam theory". International Journal of Solids and Structures, Vol 37 No 3 Oct 2000. p 535558

[15] Popescu B., Hodges D.H., Cesnik C.E.S. "Obliqueness effects in asymptotic cross-sectional analysis of composite beams". Computers and Structures, Vol 76 No 4 2000. pp. 533543

[16] Timoshenko S.P., Goodier J.N. "Theory of Elasticity". McGraw-Hill, Maiden-Head, UK, 1970

[17] Volovoi V.V. "A unifying look at the cross-sectional analyses for composite beams". Proceedings of the $42^{\text {nd }}$ AIAA/ASME/ASCE/ AHS/ASC Structures, Structural Dynamics, and Materials Conference, Seattle, WA, April 2001. AIAA Paper 2001-1404

[18] Volovoi V.V., Hodges D.H., Berdichevsky V.L,. Sutyrin V.G. "Dynamic dispersion curves for non-homogeneous, anisotropic beams with cross-sections of arbitrary geometry". Journal of Sound and Vibration, Vol 215, No 5, 3 Sept. 1998. pp.1101-1120

[19] Volovoi V.V., Hodges D.H., Berdichevskii V.L., Sutyrin V.G. "Asymptotic theory for static behavior of elastic anisotropic I-beams". International Journal of Solids and Structures, Vol 36 No 7, Mar 1999. pp. 1017-1043

[20] Wilkie W.K., Bryant R.G., High J.W., Fox R.L., Hellbaum R.F., Jalink A., Little B.D., Mirick P.H. "Low-cost piezocomposite actuator for structural control applications". Proceedings of the SPIE Sympsosium on Smart Structures and Materials, Newport Beach CA, March 5-9 2000. pp. 323-334

[21] Yu W., Hodges D.H., Cesnik C.E.S. "On Timoshenko-like Modeling of Initially Curved and Twisted Composite Beams". International Journal of Solids and Structures, Vol 39, Issue 19, Sept 2002. pp 5101-5121 